\title{
Teoria dos registros de representação semiótica em pesquisas na Educação Matemática no Ensino Superior: uma análise de superfícies e funções de duas variáveis com intervenção do software Maple
}

\author{
Registers of semiotic representation theory in mathematics \\ education researches in higher education: an analysis of surfaces \\ and two variables functions using the software Maple
}

Afonso Henriques $^{1}$ • Saddo Ag Almouloud ${ }^{2}$

\begin{abstract}
Resumo: Este artigo traz algumas reflexões motivadas pela utilização equivocada de noções da Teoria dos Registros de Representação Semiótica (TRRS), proposta por Duval, pelos estudantes e pesquisadores em suas pesquisas/palestras. Sem perda de essência e generalidade, trazemos algumas definições fundadas na proposta de Duval e utilizamos os conceitos de cálculo diferencial e integral com ênfase nas superfícies e funções de duas/três variáveis, como objetos matemáticos de referência. O objetivo é explicitar as referidas definições, na tentativa de trazer contribuições da TRRS na análise em pesquisas em Educação Matemática no Ensino Superior, além de auxiliar os estudantes na reflexão sobre o tema, discutir as técnicas instrumentais de representação do tipo de funções em questão nos diferentes registros, em especial no registro gráfico em três dimensões, utilizando o software Maple, porque o fenômeno "ver" ou "representar" objetos matemáticos em 3D é um dos entraves na aprendizagem de cálculo pela maioria dos estudantes.
\end{abstract}

Palavras-chave: Ensino superior. Educação matemática. Teoria dos registros. Representação semiótica. Signo.

\begin{abstract}
This paper presents some reflections motivated by mistakes that students and researchers have made in their lectures/research when they are using the Registers of Semiotics Representation Theory (RSRT), proposed by Duval. Without loss of generality and essence, we take some definitions from the Duval proposal and the concepts of differential and integral calculus (focused specifically on surfaces and two / three variables functions) in order to use them as mathematical objects of reference. The main goal is explain those definitions in such way to highlight RSRT contributions for the analysis of mathematics education researches practices in higher education and assist students in thinking about this topic, discussing the instrumental techniques of representation of those functions in different registers, especially for the three dimensional graphic registers using the software Maple, since the phenomenon "see" or "represent" 3D mathematical objects is one of the obstacles in the calculus learning of almost all students.
\end{abstract}

Keywords: Conversion. Treatment. Object of knowledge. Definitions. Sign.

\footnotetext{
${ }^{1}$ Universidade Estadual de Santa Cruz (UESC), Departamento de Ciências Exatas e Tecnológicas, Pós-Graduação em Educação Matemática, Ilhéus, BA, Brasil. E-mail: <henry@uesc.br>.

${ }^{2}$ Pontifícia Universidade Católica de São Paulo (PUC-SP), Programa de Estudos Pós-graduados em Educação Matemática, São Paulo, SP, Brasil.
} 


\section{Introdução}

Neste artigo, são tecidas algumas reflexões sobre a Teoria dos Registros de Representação Semiótica (TRRS) de Duval $(1988,1993,1995,1998)$ em um contexto mais geral de suas aplicações em algumas pesquisas e, mais especificamente, no estudo de superfícies e funções de duas e três variáveis. O nosso intuito neste artigo é, além de contribuir com os estudos que se inscrevem nessa teoria, auxiliar os estudantes na reflexão sobre o tema e discutir as técnicas instrumentais de representação de funções em questão nos diferentes registros, em especial no registro gráfico, utilizando o software Maple na modelagem de superfícies, porque o fenômeno "ver" ou "representar" objetos matemáticos em três dimensões é um dos entraves na aprendizagem de CDI e Geometrias pelos estudantes. A título de exemplo, basta observar os recortes das práticas efetivas de estudantes em uma das avaliações de CDI que apresentamos na sexta seção deste artigo. Além da TRRS, as nossas reflexões se apoiaram em alguns aspectos da Teoria Antropológica do Didático (TAD) de Chevallard (1992, 1999).

$\mathrm{Na}$ primeira parte do artigo, fazemos algumas reflexões motivadas pela utilização, às vezes equivocada, de certas noções relacionadas à Teoria dos Registros de Representação Semiótica, proposta por Duval, por estudantes em fase de aprendizagem, bem como por pesquisadores que a utilizam em suas pesquisas.

$\mathrm{Na}$ segunda parte, apoiando-se, em algumas definições fundadas na proposta de Duval, e utilizamos os conceitos de Cálculo Diferencial e Integral (CDI) com ênfase nas superfícies e funções de duas e três variáveis, como objetos matemáticos de referência. O objetivo é explicitar as referidas definições, na tentativa de trazer contribuições da noção de registros de representação semiótica na análise em pesquisas em Educação Matemática no Ensino Superior (IES).

$\mathrm{Na}$ terceira parte, definir-se-ão as noções de traços, curvas e superfícies de nível, e discutimos a necessidade de um estudante saber mobilizar a multiplicidade de seus conhecimentos anteriores, juntamente com as técnicas instrumentais.

Debater-se-á, na quarta parte, a relação entre a TRRS e as variáveis visuais significativas no estudo de superfícies e de curvas no espaço tridimensional. Mais especificamente, refletimos sobre as limitações das variáveis visuais, definidas por Duval, nos casos mais simples como o traçado de uma reta no plano cartesiano, no estudo de superfícies e de curvas em 3D.

$\mathrm{Na}$ quinta parte, apresentar-se-á sutilmente um recorte da produção efetiva de oito estudantes de um curso de CDI III no intuito de mostrar que a realização de certas tarefas que envolvem superfícies e curvas em 3D no ambiente papel/lápis é um desafio para eles.

\footnotetext{
${ }^{3} \mathrm{O}$ Maple é um ambiente computacional (software) para Matemática avançada que inclui ferramentas para álgebra, equações diferenciais, análise matemática, matemática discreta, cálculo gráfico e numérico, etc. Ele é também uma linguagem de programação que utiliza uma biblioteca de funções e operações integradas. Foi desenvolvido por um grupo de pesquisadores da Universidade Waterloo, Canadá. A sua primeira versão data de 1980.
} 


\section{A Teoria dos registros de representação semiótica: algumas utilizações problemáticas do termo "conversão"}

A Teoria de Registros de Representação Semiótica (TRRS), proposta por Duval (1995), é uma das abordagens teóricas que vem encontrando espaço expressivo nas pesquisas em Educação Matemática no Brasil. O autor trouxe contribuições significativas no campo educativo, tanto no ensino quanto na pesquisa, tornando explícitos os conhecimentos que até então eram ou ainda são tratados implicitamente, sem que sejam evocados. Quando as noções não são evocadas, elas podem passar despercebidas no processo de aquisição de conhecimentos, deixando vazios didáticos ${ }^{4}$ na conceitualização. Por mais simples que sejam, as noções devem ser evocadas durante o seu tratamento (cf. Definição 5 mais adiante) para garantir a consolidação da aprendizagem. Nesse tratamento, dois termos não devem ser confundidos, a saber: objeto e representação. Para Duval (1998, p. 140), "as relações existentes entre os dois termos são as noções centrais para toda a análise do conhecimento". A representação de um objeto e a conversão de representações entre registros, por exemplo, são comuns nas práticas do professor de Matemática em sala de aula, quando este pretende fazer com que os seus alunos compreendam uma determinada noção de difícil entendimento no registro no qual o objeto foi inicialmente apresentado. No momento em que o professor realiza essa conversão, não implica, necessariamente que ele queira reforçar a estreita relação existente entre os registros que mobilizou. Essa reflexão vai ao encontro com a ideia de Duval (1993, p. 37), quando o autor escreve:

Existe uma palavra que é ao mesmo tempo importante e marginalizada em Matemática, é a palavra "representação": ela é frequentemente empregada sob sua forma verbal, "representar". Uma escrita, uma notação, um símbolo, representam um objeto matemático: um conjunto, uma função, um vetor [...] o que significa dizer que os objetos matemáticos não devem ser confundidos com suas representações. Toda confusão implicará uma perda de compreensão e, consequentemente, os conhecimentos adquiridos se tornam inutilizáveis no seu contexto de aprendizagem. A distinção entre um objeto e sua representação é, portanto, um ponto estratégico na compreensão da matemática.

Assim, o professor que pretende fazer com que os seus alunos aprendam Matemática, sob diferentes pontos de vista, não deve, simplesmente, tratá-la sem evocar o importante papel exercido pelos diferentes registros que ele mobiliza em função dos objetos matemáticos a representar/ensinar. Duval (1993, p. 38) acredita que "existe um paradoxo cognitivo do pensamento matemático: de um lado, a apreensão dos objetos matemáticos pode ser apenas uma apreensão conceitual e, de outro lado, só por meio de representações semióticas é que uma atividade sobre objetos matemáticos é possível". Com base nessa concepção, podemos apresentar a seguinte definição:

Definição 1: Representação semiótica é uma representação de uma ideia ou um objeto do saber, construída a partir da mobilização de um sistema de sinais. Sua significação é determinada, de um lado, pela sua forma no sistema semiótica e de outro lado, pela referência do objeto representado.

\footnotetext{
${ }^{4}$ Vazio didático é a existência de saberes em torno de um objeto de conhecimentos que não são mobilizados pelo sujeito e compromete o ensino correspondente, bem como a realização efetiva de situações-problemas ou tarefas concernentes.
} 
Um enunciado em língua materna, uma fórmula algébrica, um gráfico de uma função ou uma figura geométrica, um conjunto de números, por exemplo, são representações semióticas que revelam sistemas semióticos diferentes, com diferentes signos.

A literatura tem revelado diversos significados do termo signo. Muitos deles nem sempre esclarecem, de fato, o que se pretende colocar em cena, no estudo em questão, instalando ainda mais equívocos, particularmente nos estudos de objetos matemáticos que já são complexos por diversos estudantes. Esta é uma questão extremamente relevante, na medida em que todo raciocínio é relacionado a um signo de algum tipo. Para Peirce (2005, p. 46, grifo do autor):

Um signo, ou representâmen, é aquilo que, sob certo aspecto ou modo, representa algo para alguém. Dirige-se a alguém, isso cria na mente dessa pessoa um signo equivalente, ou talvez um signo mais desenvolvido. Ao signo assim criado denomino interpretante do primeiro signo. O signo representa alguma coisa do seu objeto. Representa esse objeto não em todos os seus aspectos, mas com a referência a um tipo de ideia que eu, por vezes, denominei de fundamento do representâmen.

Amparados em Peirce e na proposta de Duval sobre os estudos defendidos aqui, em especial conforme os registros destacados na Figura 1, apresentamos a seguinte definição para signo, visando aos trabalhos possíveis nesses registros.

Definição 2: Um signo é um sinal mobilizado por alguém (sujeito) capaz de permitir-lhe identificar um sistema ou registro de representação semiótico (cf. Definição 3), como as regras linguísticas ou gramaticais na língua materna, as propriedades ou escritas algébricas para o registro algébrico, as figuras geométricas (pontos, segmentos/ retas/curvas, planos e superfícies) para o registro gráfico, os números, as operações aritméticas, para o registro numérico e, de um modo geral as regras de conformidade ${ }^{5}$.

Assim, dentre os registros de representação que se podem pensar na Educação Matemática, desde a Educação Básica ao Ensino Superior, quatro são predominantes. A Figura 1 traz um esquema que retrata as definições 1 e 2 como forma de contribuir na reflexão sobre um objeto e suas possíveis representações nos quatro diferentes registros.

Figura 1. Possíveis registros de representação de um objeto matemático

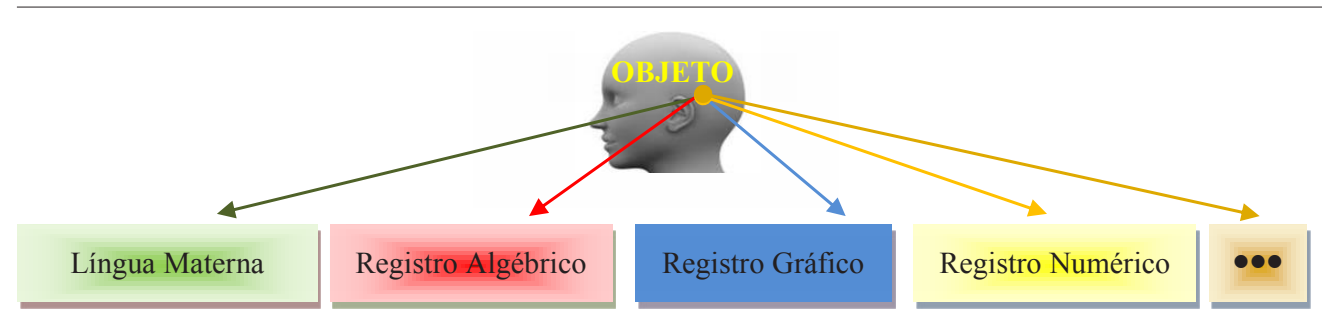

Fonte: Produção dos autores.

\footnotetext{
${ }^{5}$ Regras a respeitar na formação de uma representação semiótica, tais como regras gramaticais quando se trata de línguas maternas, regras de representação gráfica, regras de cálculos numéricos.
} 
Teoria dos registros de representação semiótica ...

O tratamento dos objetos matemáticos depende, portanto, das possibilidades de suas representações. Mas, o que é um registro de representação?

Definição 3: Um registro de representação é um sistema dotado de signos que permitem identificar uma representação de um objeto de saber.

Conforme esquematizado na Figura 1, um dado objeto de saber pode ser representado em diferentes registros dotados de diferentes signos. Pela definição 3, o registro é o sistema no qual ocorre ou se realiza a representação de um dado objeto, externando, assim, o objeto não ostensivo (ideia, noção, conceito) pensado pelo sujeito. Pois, como afirma Chevallard (1992), os objetos não ostensivos só são acessíveis a partir dos objetos ostensivos associais.

Quando um indivíduo pensa em um objeto (ideia, noção, conceito, etc.), apenas ele tem acesso a esse objeto naquele instante, pois pensar é faculdade do sistema mental. Tornar tal objeto acessível aos outros indivíduos implica evocá-lo, externá-lo por "gestos" ou por meio da sua representação em um registro. Este último é um sistema estático dotado de signos, enquanto que a representação ou as representações feitas nesse registro são dinâmicas, no sentido de que podem sofrer tratamentos no próprio registro ou conversões entre diferentes registros. Afirmamos, por conseguinte, que não se converte um registro, pois este tem a função de acomodar as representações de objetos em jogo. Duval (1995) distingue três atividades cognitivas, fundamentais, ligadas aos registros de representações traduzidos neste artigo como definições (de 4 a 6) importantes nessa abordagem:

Definição 4: A formação de uma representação semiótica é baseada na aplicação de regras de conformidade e na seleção de certas características do conteúdo envolvido.

Por exemplo, a composição de um texto, construir uma figura geométrica, elaborar um esquema, escrever uma fórmula, descrever o domínio de uma função, etc.

Definição 5: O tratamento de uma representação é a transformação desta em outra representação no mesmo registro no qual foi formada. O tratamento é, portanto, uma transformação interna num registro.

Por exemplo, o cálculo é uma forma de tratamento próprio das escritas simbólicas (cálculo numérico, cálculo algébrico, cálculo de limite de uma função, cálculo integral de uma função, cálculo proposicional...).

Definição 6: A conversão de uma representação é a transformação desta representação em uma representação de outro registro.

Por exemplo, a tradução de um texto em uma ou mais expressões algébricas correspondentes é uma conversão da representação destas expressões da língua materna para o registro algébrico. A conversão é, portanto, uma atividade cognitiva diferente e independente do tratamento.

A escolha de um registro de representação adequado para externar os conceitos de um objeto de saber pode favorecer o tratamento. No entanto, Duval (1995) afirma que dispor de 
vários registros de representação não é suficiente para garantir a compreensão. Uma segunda condição é necessária: a coordenação de representações formuladas em registros distintos.

Definição 7: A coordenação é a manifestação da capacidade do indivíduo em reconhecer a representação de um mesmo objeto, em dois ou mais registros distintos.

A coordenação aparece como a condição fundamental para todo tipo de aprendizagem. Retomando a Figura 1, reforça-se, conforme a Figura 2, em concordância com a Definição 7, que os registros são sistemas inertes que acomodam as representações de objetos de saberes que, por sua vez, são dinâmicas, na medida em que elas podem, com base nas Definições 5 e 6 , sofrer transformações no mesmo ou entre diferentes registros.

Figura 2. Conversão e coordenação de representações de um objeto entre registros

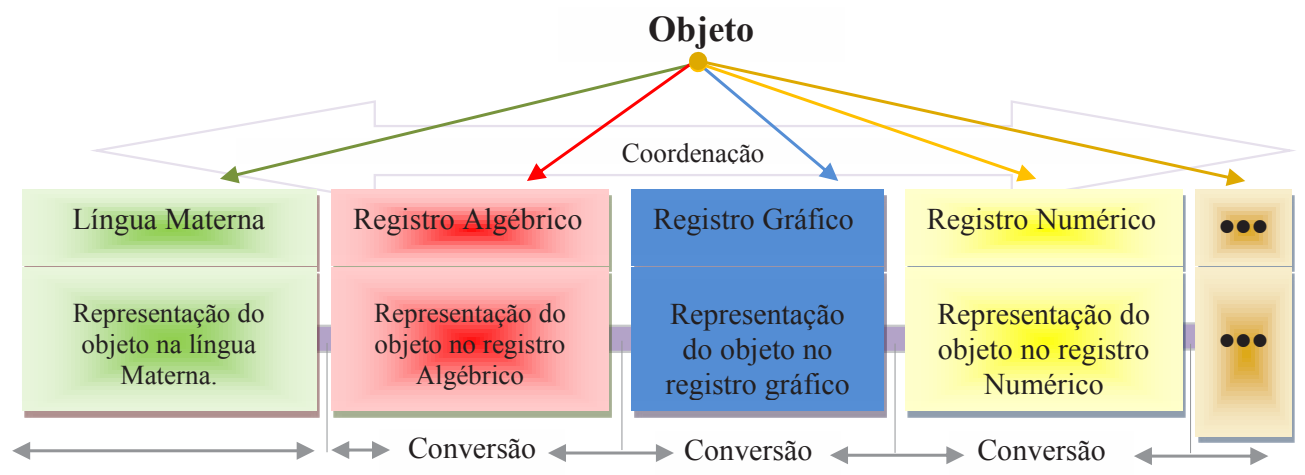

Fonte: Produção dos autores.

A Definição 6 é, muitas vezes, empregada de forma errônea ou inconclusiva (com vazios didáticos) pelos estudantes em fase de aprendizagem / utilização da perspectiva teórica Noção de Registros de Representação Semiótica (NRRS) proposta por Duval, bem como por pesquisadores em nível avançado, principalmente em artigos publicados ou dissertações prontas. É frequente encontrar nas análises apresentadas pelos pesquisadores descrições ou discursos do tipo:

Dos alunos que optaram por utilizar a conversão da forma algébrica para a forma tabular como parte da resolução da atividade, mais da metade apresentou erros de cálculos na manipulação da expressão algébrica. (DELGADO, 2010, p. 71, grifos nossos).

Ao analisarmos como o Objeto explora os tratamentos e as conversões, verificamos que a maioria das atividades exige o tratamento gráfico $[\ldots]$ a 
Teoria dos registros de representação semiótica ...

fim de que o aluno consiga realizar a conversão do registro gráfico para o simbólico. (BONOTO; SOARES; MARTINS, 2010, p. 13, grifos nossos).

De modo mais específico, pretende-se avaliar os fenômenos didáticos ocorridos na resolução de problemas envolvendo a conversão do registro gráfico de uma função afim para o algébrico e vice-versa. (SILVA, 2005, p. 31, grifos nossos).

A conversão do registro algébrico para o registro gráfico, na maioria das situações de Modelagem, não apresentou dificuldades para os alunos [...] (VERTUAN; SILVA; ALMEIDA, 2009, p. 4)

Como se pode notar nessas descrições, a conversão é versada ao registro e não à representação. Além disso, em muitas vezes, o objeto representado não é evocado na conversão. Acreditamos ser possível reverter esse quadro, pois não se converte o registro, mas a representação do objeto em questão de um registro para outro. Para compreender melhor que essas descrições expressam interpretações equivocadas, é suficiente fazer uma analogia, considerando as duas janelas (algébrica e geométrica) do softwrare GeoGebra, como registros distintos, o objeto sendo, por exemplo, a Função Quadrática, sendo a representação de uma função quadrática específica, inicialmente formulada na janela algébrica, e a conversão sendo a transformação ou a representação gráfica dessa função na janela geométrica. Logo, quem se transforma ou muda de registro não é a janela, mas a representação formulada nessa janela. Assim, insiste-se em afirmar, com base na Definição 6, que não se podem converter registros, mas as representações de objetos neles formuladas.

\section{Registros no estudo de superfícies, funções de duas e três variáveis na Educação Matemática no Ensino Superior}

Para Chevallard (1992), um objeto de saber existe se um indivíduo ou uma instituição o reconhece como existente. Suponhamos então, que um indivíduo reconhece um determinado objeto matemático tal como as superfícies ou funções de duas variáveis. É possível que esse seja, então, capaz de evocar ou definir esses objetos na língua materna, conforme segue:

Definição 8: Uma Superfície $S$ no registro gráfico é o conjunto de todos os pontos $(x, y, z)$ do espaço tridimensional que satisfazem a equação $F(x, y, z)=0$ no registro algébrico.

Definição 9: Uma função de duas variáveis é uma correspondência que associa a cada par ordenado do seu domínio, que seja um subconjunto D do plano (plano- $x y$, plano- $x z$ ou plano-yz) exatamente um número real de um subconjunto I da reta (eixo-x, eixo-y ou eixo-z), respectivamente.

Sendo $\mathscr{D}$ um subconjunto de um plano, que seja o plano-xy e $f$ uma função de duas variáveis definida em $\mathcal{D}$, então podemos considerar $z=f(x, y)$ como sendo a equação que, no registro gráfico, consiste na Superfície $S$. Além disso, pode-se escrever $f(x, y)-z=0$. Fazendo 
$F(x, y, z)=f(x, y)-z$, a equação de superfície pode ser representada por $F(x, y, f(x, y))=0$. Nestas condições, pode-se apresentar outra definição.

Definição 10: A Superfície $S$ de equação $F(x, y, z)=0$ no registro algébrico é o gráfico da função $f$, tal que $z=f(x, y)$.

Assim, diz-se que a língua materna é um registro de representação no qual a noção ou a Definição 9 de funções de duas variáveis, por exemplo, é formulada. Essa definição pode ser representada em símbolos matemáticos, realizando-se, assim, sua conversão, da língua materna para o registro algébrico, que identificamos por Alg.01, constituindo um primeiro elemento da sequência de representações no registro algébrico que doravante aparecerão neste artigo, onde utilizamos o símbolo $\mathbb{R}$ para representar o conjunto dos números reais.

$$
\begin{aligned}
\mathscr{D} \subset \mathbb{R} \times \mathbb{R} & \rightarrow I \subset \mathbb{R} \\
(x, y) & \rightarrow f(x, y)
\end{aligned} \quad \text { ou } \quad f: \mathscr{D} \subset \mathbb{R} \times \mathbb{R} \rightarrow I \subset \mathbb{R} \quad \text { Alg.01 }
$$

Nesse caso, diz-se que o objeto função de duas variáveis está representado no registro algébrico, no qual se escreve $z=f(x, y)$ com $z \in I$. O símbolo $I$ denota o conjunto imagem de $f$ e $\mathbb{R}$ o seu contradomínio. Se, por exemplo, $f$ é a função de duas variáveis dada por $f(x, y)=$ $1+x^{2}+y^{2}$, considerada em Swokowski (1994, p. 432), dir-se-á que o domínio $\mathscr{D} \subset \mathbb{R} \times \mathbb{R}=\mathbb{R}^{2}$ é o plano- $x y$, que é representado analiticamente pelo conjunto $\mathscr{D}$ (Alg.02) no registro algébrico.

$$
\mathcal{D}=\left\{(x, y) \in \mathbb{R}^{2} ;-\infty<x<\infty,-\infty<y<\infty\right\}
$$

$\mathcal{D}$ está representado no registro algébrico, pois, em sua formação, mobilizam-se características e regras de conformidade (cf. Definição 3), tais como as variáveis matemáticas $x$ e $y$, os pares ordenados, pertinência, potência, sinais de subtração, de adição e desigualdades convenientemente. Isso ocorre também na Alg.01. O conjunto imagem de $f$ é o subconjunto $I=1+z_{+} \subset \mathbb{R}$ que é representado analiticamente por:

$$
I=\{z \in R ; 1 \leq z<\infty\}
$$

no registro algébrico. Particularmente a função dada por:

$$
f(x, y)=1+x^{2}+y^{2}
$$

é uma representação, no registro algébrico, do objeto matemático denominado parabolóide. A modelagem ou a conversão da representação algébrica dessa função para sua representação no registro gráfico não é uma prática evidente para muitos estudantes de cursos de Cálculo Diferencial e Integral (CDI). Além disso, a maioria dos livros didáticos que analisamos e que contêm o babitat ${ }^{6}$ de tipo de tarefas (no sentido praxeológico) (CHEVALLARD, 1999) que

\footnotetext{
${ }^{6}$ Habitat é o lugar de vida e o ambiente conceitual de um objeto do saber (CHEVALLARD, 1992).
} 
envolvem a superfície de equação $z=1+x^{2}+y^{2}$, entre outras, apresenta-a como "algo que cai do céu", na medida em que os autores simplesmente escrevem "a figura ao lado mostra...", sem que sejam explicadas as técnicas que permitem a modelagem e a visualização de tal superfície parabólica. As Figuras 3 e 4 ilustram essa superfície no registro gráfico.

Figura 3. Parabolóide truncado por quatro planos verticais

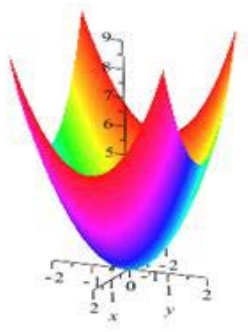

Fonte: Produção dos autores.
I1. with(plots)

I2. $f:=(x, y)->1+x^{\wedge} 2+y^{\wedge} 2$

I3. $\operatorname{plot} 3 \mathrm{~d}(f(x, y), \mathrm{x}=-2 . .2, \mathrm{y}=-2 . .2$, color $=x$ )

I4.plot3d([r. $\cos (t), r \cdot \sin (t), f(r$. $\cos (\mathrm{t}), \mathrm{r} \cdot \sin (\mathrm{t})), \mathrm{r}=0 . .2, \mathrm{t}=0 . .2$. $\mathrm{Pi}$, color $=\mathrm{t}$ )

A instrução I3 retoma a Figura 3 e a instrução I4 a Figura 4. E possível notar que os dois gráficos são visualmente diferentes. Ambos são truncados por planos cujas representações não estão visíveis.
Figura 4. Parabolóide truncado por um plano horizontal

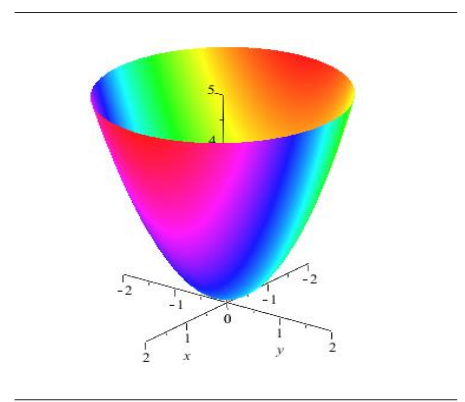

Fonte: Produção dos autores.

Vale sublinhar que essas Figuras 3 e 4 não são representações gráficas da função $f$, mas representações da função $f$ no registro gráfico, ou simplesmente gráficos da função $f$. Salienta-se ainda que, para o Dicionário Brasileiro Globo (FERNANDES; LUFT; GUIMARÃES, 1990), representação é o “ato ou efeito de representar". O termo representação gráfica é conceitualmente empregado de forma equivocada em Matemática, pois a representação gráfica é o processo que conduz à visualização de um representante da função no registro gráfico.

Ambos os gráficos das Figuras 3 e 4 representam o mesmo objeto matemático (o parabolóide). Mas, por que o primeiro mostra quatro "pontas" e o segundo não? Quando essa questão é colocada aos estudantes em curso de CDI, eles não conseguem imediatamente explicar a diferença, pois os referidos planos, fatores dessa diferença, estão invisíveis nesses gráficos. Contudo, aplicando a Definição 5, pode-se compreender melhor os fenômenos inerentes. $\mathrm{Na}$ Figura 3, tais planos são determinados lateralmente pelos extremos ou fronteira do domínio da função representado no Maple e superiormente pelo maior valor assumido pela função nesse domínio. Na Figura 4, o plano que trunca esse objeto é a superfície de equação $z=5$. Sublinha-se que ambas as figuras não mostram exatamente o objeto parabolóide (da função em questão), mas um representante dessa função no registro gráfico, sobre os domínios escolhidos que são representados analiticamente por:

$$
\mathscr{D}=\left\{(x, y) \in \mathbb{R}^{2} ;-2 \leq x \leq 2,-2 \leq y \leq 2\right\}
$$

para a Figura 3, e analiticamente por:

$$
\mathcal{D}=\left\{(r, \theta) \in \mathbb{R}^{2} ; 0 \leq r \leq 2,0 \leq \theta \leq 2 \pi\right\}
$$


para a Figura 4. Aplicando a técnica instrumental Crivo-Geométrico (HENRIQUES, 2006), pode-se recuperar a parte de cada plano em questão que, juntamente com as superfícies de Figuras 3 e 4, delimitam os sólidos representados nas Figuras 5 e 6.

Figura 5. Crivos dos planos que contêm a fronteira do domínio de $f$, do plano superior e do gráfico de $f$.

Fonte: Produção dos autores.

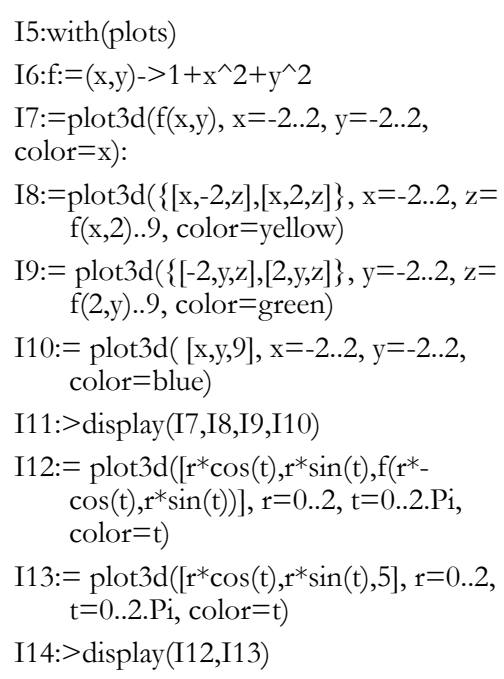

Figura 6. Crivo do gráfico de $f$ e do plano superior de equação z=5 no domínio de $f$.

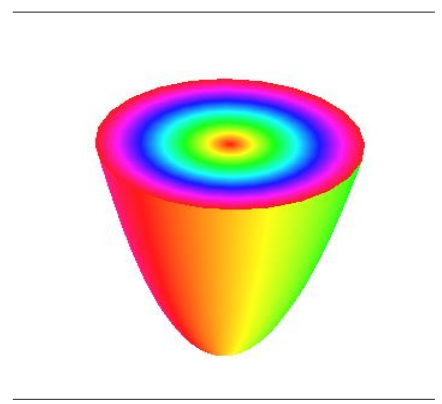

Fonte: Produção dos autores.

As instruções de I5 a I11 são técnicas instrumentais que retornam o gráfico da Figura 5 e as instruções de I12 a I14, o gráfico da Figura 6. Sublinhamos que as relações possíveis existentes entre uma função de duas variáveis e o seu domínio, no registro gráfico, são muitas vezes discriminadas no ensino da Matemática na Educação Superior, que privilegia as mesmas relações no registro algébrico. Com efeito, os estudantes manifestam dificuldades em estabelecer essas relações.

É possível, por intermédio das Figuras de 7 a 10, notar que as referidas relações, quando evocadas e exploradas explicitamente, podem favorecer a compreensão do conceito de função relativo a domínio. Assim, é essencial reforçar que as regiões dadas nas Figuras 7 e 10 são resultantes de conversões das representações, do objeto domínio da função, representadas no registro algébrico em Alg.05 e 06 para o registro gráfico.

As Figuras 7 e 10 ilustram também o conjunto imagem associado à visualização de cada gráfico de $f$, cujos correspondentes no registro algébrico são, respectivamente, as representações dos intervalos ou conjuntos dos números reais identificados por Alg.07 e 08.

$$
\begin{aligned}
& I=\{z \in R ; 1 \leq z \leq 9\} \\
& I=\{z \in R ; 1 \leq z \leq 5\}
\end{aligned}
$$

Estendem-se as análises sobre registros para o estudo de objetos que intitulam o tópico seguinte a partir da função que considerada anteriormente para, em seguida, ponderar as variáveis visuais significativas no estudo de superfícies e curvas em $3 \mathrm{D}$ e, mais adiante, apresentar uma análise concisa das práticas de estudantes em CDI. 
Figura 7. Região que consiste no Domínio do crivo do gráfico de $f$ no sistema de coordenadas cartesianas.

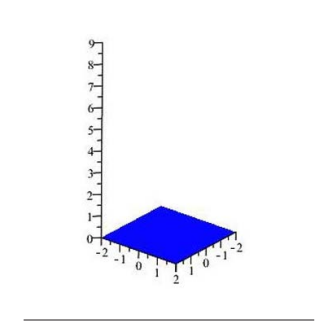

Fonte: Elaborada pelos autores.
Figura 8. Sólido delimitado pelos crivos: do gráfico de $f$, dos planos emanados da fronteira do domínio e do plano superior de equação $z=9$, assim como a Figura 7.

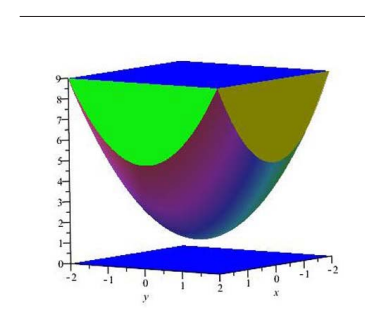

Fonte: Elaborada pelos autores.
Figura 9. Sólido delimitado pelos crivos do gráfico de $f$ e do plano superior de equação $\mathrm{z}=5$, assim como a Figura 10.

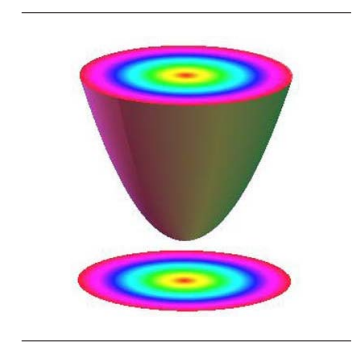

Fonte: Elaborada pelos autores.
Figura 10. Região que consiste no Domínio do crivo de $f$ em coordenadas polares.

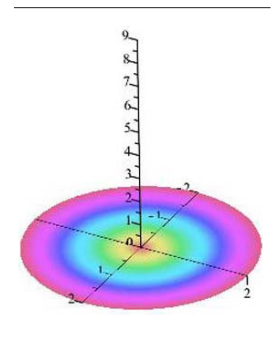

Fonte: Elaborada pelos autores.

\section{Traços, curvas e superfícies de nível}

Iniciamos esta parte do artigo apresentando o seguinte conceito:

Definição 11: O traço do gráfico de uma função é a curva determinada pela interseção desse gráfico com um plano paralelo ao plano que contém o domínio de definição dessa função na língua materna.

Se o gráfico de uma função é um plano, então o referido traço ou curva é uma reta. Assim, pode-se afirmar que toda reta é uma curva de curvatura nula. Suponhamos que a função seja de duas variáveis, como aquela considerada anteriormente, isto é, a função com a lei de formação indicada em Alg.09:

$$
f(x, y)=1+x^{2}+y^{2}
$$

Nesse caso, o referido plano tem como equação $z=z_{0} \operatorname{com} z_{0} \in I$ sendo $I$ o mesmo que foi definido em Alg.03. A conversão da Definição11 para o registro gráfico, considerando o caso específico de Alg.09, resulta nas representações ilustradas nas Figuras 11 e 12 considerando nove planos. A projeção dos traços da Figura 12 para o plano-xy constitui as curvas de níveis da função $f$ correspondentes aos traços em jogo. No caso em análise, tais curvas são circunferências concêntricas de centro na origem de sistema de coordenadas, conforme se mostra na Figura 13. No curso de cálculo, os estudantes apresentam dificuldades manifestadas pela ausência de competências nas descrições de esboços que realizam no registro gráfico. Além disso, a escrita ou descrição sobre o que fazem não é motivada nas práticas dos estudantes de cursos de ciências exatas, em particular nos cursos de Matemática (seja Bacharelado ou Licenciatura), nos quais a maioria dos estudantes se prende na realização de contas. 
Figura 11. Planos e o gráfico de $f$.

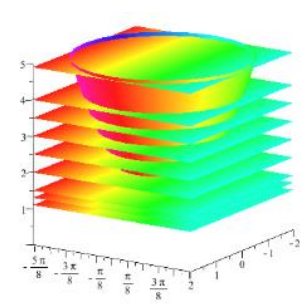

Fonte: Produção dos autores.
Figura 12. Traços do gráfico de $f$.

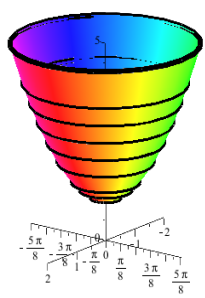

Fonte: Produção dos autores.
Figura 13. Curvas de níveis.

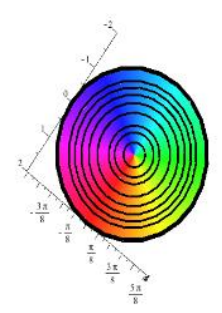

Fonte: Produção dos autores.

Esses modelos são cobertos pelas instruções implementadas no Maple na conversão das representações dos planos e da função em jogo entre registros, e exigem do sujeito um tratamento inicial desses objetos na língua materna (descrição) e no registro algébrico. Nessa ocasião, o sujeito deve necessariamente mobilizar a multiplicidade de seus conhecimentos anteriores, juntamente com as técnicas instrumentais. Tais técnicas são laboriosas. Isso, talvez, explique a sua ausência nos livros didáticos ${ }^{7}$ analisados, quando os autores simplesmente dizem "a figura ao lado mostra...", deixando-se essa tarefa a cargo do professor.

Além da noção de curvas, propôs-se a estudar as superfícies de nivel que repousam sobre o conceito de funções de três variáveis, começando pela seguinte definição:

Definição 12: Se F é uma função de três variáveis, chamam-se superfícies de nivel de $F$ ao resultado das representações das superfícies de equações $F(x, y, z)=k$ no registro gráfico, nas quais ké uma constante real.

A reescrita da lei de formação da função $f$ dada por:

$$
f(x, y)=1+x^{2}+y^{2}
$$

pela equação identificada por Alg. 11:

$$
z=1+x^{2}+y^{2}
$$

é um resultado da transformação ou tratamento da representação de $f$ no registro algébrico. Assim, pode-se, novamente, por tratamento algébrico, obter-se:

$$
z-1-x^{2}-y^{2}=0
$$

\footnotetext{
${ }^{7}$ Para esta análise, o leitor é convidado a consultar a seção 5.2, de Henriques, Nagamine e Nagamine (2012).
} 
Representando o primeiro membro de Alg.12 por $F(x, y, z)$ tem-se:

$$
F(x, y, z)=z-1-x^{2}-y^{2}
$$

Pela Definição $11, F(x, y, z)=k$ é a equação de uma superfície de nível, onde k é um número real. $k$ assim definido é uma variável didática que interfere no deslocamento de cada superfície de nível ao longo do eixo-z. Pois quando $k$ assumindo diferentes valores modifica a situação (superfície). Na Figura 14 ilustra-se algumas superfícies de nível de $F(x, y, z)=z-1-x^{2}-y^{2}$ no registro gráfico a parir das instruções implementadas no software Maple, para $k=1 ; 2 ; 3$ e 4.

Figura 14. Superfícies de nível.
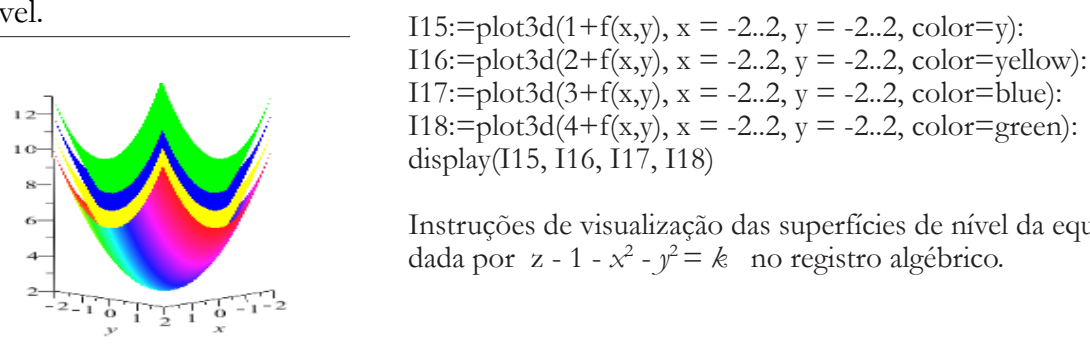

Instruções de visualização das superfícies de nível da equação dada por $z-1-x^{2}-y^{2}=k$ no registro algébrico.

Fonte: Produção dos autores.

A conversão das instruções (I15 a I18) para o registro gráfico, por mediação do comando plot3d e suas sintaxes "plot3d(exp, $x=a . . b, y=c . d,\langle o p c \tilde{c} e s\rangle)$ ", revela claramente que o domínio de representação de cada superfície de nível é uma região quadrada que já foi tratada anteriormente (cf. Alg.05).

Para compreender melhor essa conversão, é necessário que o sujeito mobilize as variáveis visuais significativas que discutiremos no item 5 deste texto.

\section{Registros e variáveis visuais significativas no estudo de superfícies e curvas em 3D}

A noção de variáveis visuais foi destacada também por Duval (1988) em um dos seus trabalhos intitulado Gráficos e equações: articulação de dois registros, no qual o autor sublinha que

a leitura de representações gráficas pressupõe a discriminação das variáveis visuais pertinentes e a percepção das variações correspondentes das expressões algébricas. Esta leitura é um procedimento de interpretação global que supõe uma atitude contrária à prática de associar um ponto a um par ordenado. (DUVAL, 1988, p. 235, tradução nossa) 
No referido trabalho, o autor distingue três tipos de tratamentos de gráficos, de acordo com o procedimento adotado:

- Ponto a ponto;

- Extensão de tracos já efetuados;

- Interpretação global de propriedades das figuras.

Ele propõe esse último como o procedimento sistemático para destacar as variáveis visuais a serem consideradas na interpretação/tratamento e explica que, em geral, as representações gráficas são introduzidas e definidas pelo primeiro procedimento, que consiste em associar um ponto a um par ordenado e vice-versa.

Sublinhamos que, no ambiente papel/lápis, esse tratamento funciona bem nos casos mais simples como o traçado de uma reta no plano cartesiano e é limitado a valores particulares referentes aos pontos marcados no plano cartesiano. Mesmo assim, também pode suscitar dificuldades nas práticas efetivas de estudantes na relação com a representação gráfica de funções de uma variável.

Logo, recorrer ao procedimento ponto a ponto torna-se improdutivo, sobretudo nas tarefas que exigem a visualização de objetos matemáticos em 3D, uma vez que, nessa dimensão, perdem-se algumas variáveis visuais. Assim, a prática sistemática do procedimento ponto a ponto não favorece o procedimento de interpretação global. Portanto, concordamos com o autor quando elege a "interpretação global de propriedades das figuras" como o procedimento sistemático para destacar as variáveis visuais a serem consideradas na interpretação de situações em jogo.

Particularmente, a articulação entre a função definida por $f(x, y)=1+x^{2}+y^{2}$ de $R^{2}$ em $R$ utilizada neste artigo com o seu correspondente no registro gráfico tem suscitado dificuldades nas práticas de estudantes.

Essas dificuldades se manifestam na tentativa de aplicação do primeiro procedimento ponto a ponto. Pois, como já sublinhado anteriormente, ver objetos no espaço 3D não é uma tarefa fácil. Esse fato pode ser notado nos recortes dos manuscritos de estudantes para os quais um professor de CDI III da Universidade Estadual de Santa Cruz (UESC), na Bahia, propõe a tarefa reproduzida no Quadro 1, no segundo semestre de 2014 (detalhar-se-á, mais adiante, sobre o professor e os estudantes).

A avaliação desse professor se inscreve no estudo de funções com valores vetoriais, que, na proposta dessa disciplina na referida Instituição, ocorre depois do estudo de Derivadas Parciais e de Integrais Múltiplas no qual os estudantes têm relações com as noções de funções de várias variáveis e representações gráficas de funções em três dimensões. Analisando o habitat do assunto dessa avaliação, é notável que nele predomina o tipo de tarefas que parte da subtarefa $t 2(a)$. Entende-se, nessa avaliação, que o professor, pretendia estabelecer relações entre os conhecimentos mobilizados pelos estudantes nos estudos dos objetos anteriores, já citados, com os conceitos de funções vetoriais, explorando, assim, a noção de curvas parametrizadas a partir de uma curva resultante da interseção de duas superfícies. Observa-se que essa ideia de relacionar/articular conceitos, aparentemente diferentes, como esses que estão em jogo aqui é ausente nos livros de CDI a que nós autores deste temos acesso no Ensino da Matemática na Educação Superior. 
Quadro 1. Reprodução do enunciado de uma avaliação de estudantes de exatas no CDI III

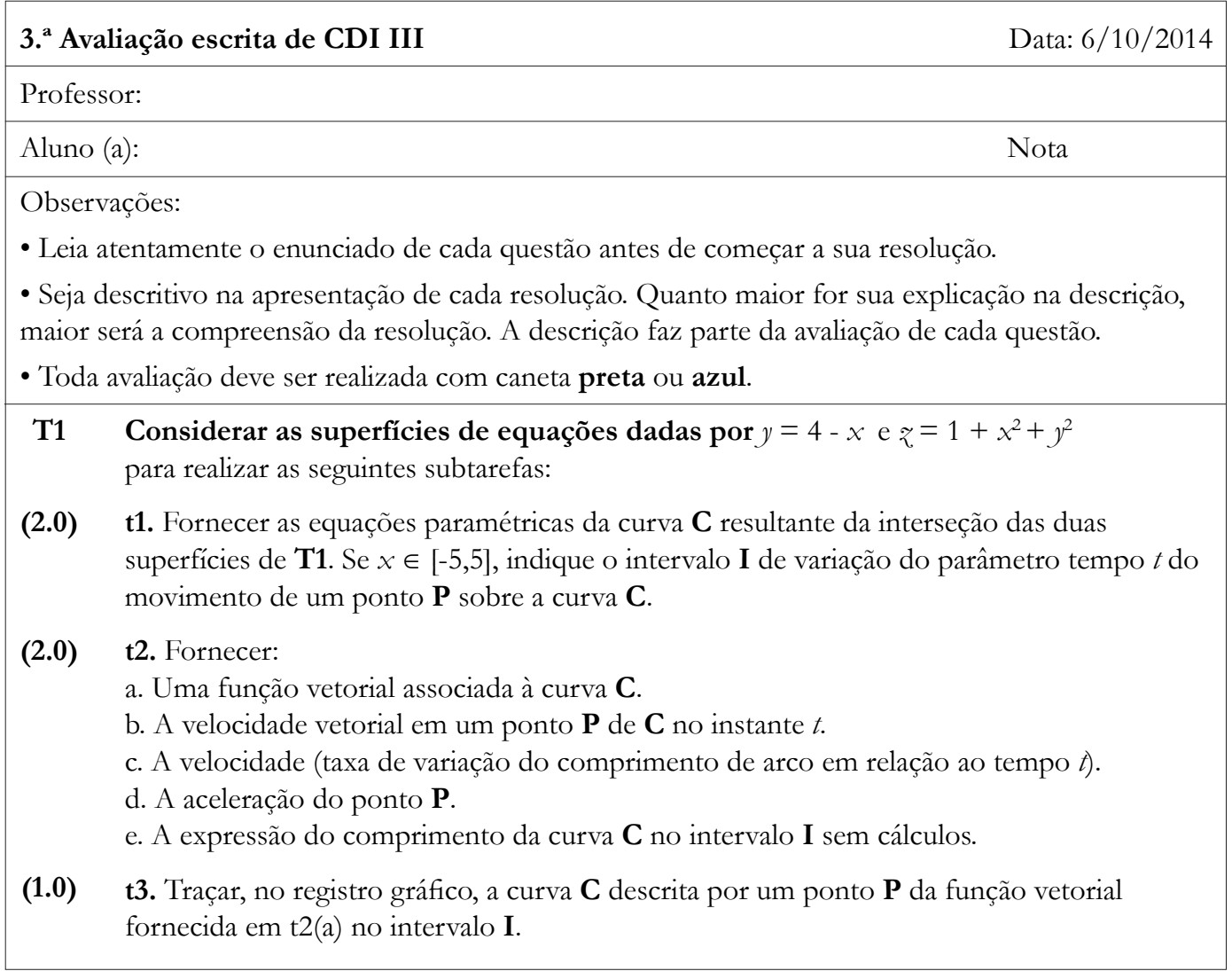

Fonte: Avaliações de um Professor em um curso de CDI III na UESC (BA).

A segunda equação da tarefa T1 dessa avaliação é clássica nos cursos de CDI e é a mesma utilizada neste artigo desde Alg.04. Para o estudante apresentar as equações paramétricas requeridas em $t 1$ ele deve, inicialmente, utilizar a Definição 5. Para isso, é suficiente substituir a equação Alg.14 na equação de Alg.15. Essa substituição corresponde, no registro gráfico, com o fenômeno de interceptar as duas superfícies em jogo.

$$
\begin{array}{lr}
y=4-x & \text { Alg.14 } \\
z=1+x^{2}+y^{2} & \text { Alg.15 }
\end{array}
$$

Elegendo a variável y para a citada substituição, obtém-se assim a equação:

$$
z=1+x^{2}+(4-x)^{2} \quad \text { ou equivalentemente } \quad z=2 x^{2}-8 x+17 \quad \text { Alg.16 }
$$


que é a equação da curva de interseção do plano dado em Alg.14 com a superfície parabólica de equação em Alg.15. Bem entendido que essa curva está contida tanto no plano (Alg.14) quanto no parabolóide (Alg.15). Assim, fazendo $x=t$, espera-se que o estudante forneça as seguintes equações paramétricas:

$$
C:\left\{\begin{array}{l}
x=f(t) \\
y=g(t), t \in I \\
z=h(t)
\end{array} \Rightarrow C:\left\{\begin{array}{l}
f(t)=t \\
g(t)=4-t \\
h(t)=2 t^{2}-8 t+17
\end{array}, t \in I=[-5,5]\right.\right.
$$

nas quais $f, g$ e $h$ são funções escalares. De posse desse resultado, o estudante deve ser capaz de realizar $t 2$ e todas as suas subtarefas, nas quais se espera a função vetorial dessa curva C que se denota por $\mathrm{r}($ ) conforme a sua representação, fornecida em Alg.18.

$$
\overrightarrow{\mathrm{r}}(t)=f(t) \mathrm{i}+g(t) \mathrm{j}+h(t) \mathrm{k}, t \in I \Rightarrow \overrightarrow{\mathrm{r}}(t)=t \mathrm{i}+(4-t) \mathrm{j}+\left(2 t^{3}-8 t+17\right) \mathrm{k}, t \in I=[-5,5] \text { Alg.18 }
$$

No registro gráfico, tem-se as representações correspondentes, por conversão (Definição 6) que apresentamos na Figura 15.

Figura 15. Visualização da curva parametrizada em (Alg.17) correspondente à interseção do parabolóide de equação dada em (Alg.15) com o plano de equação dada em (Alg.14) no registro gráfico.

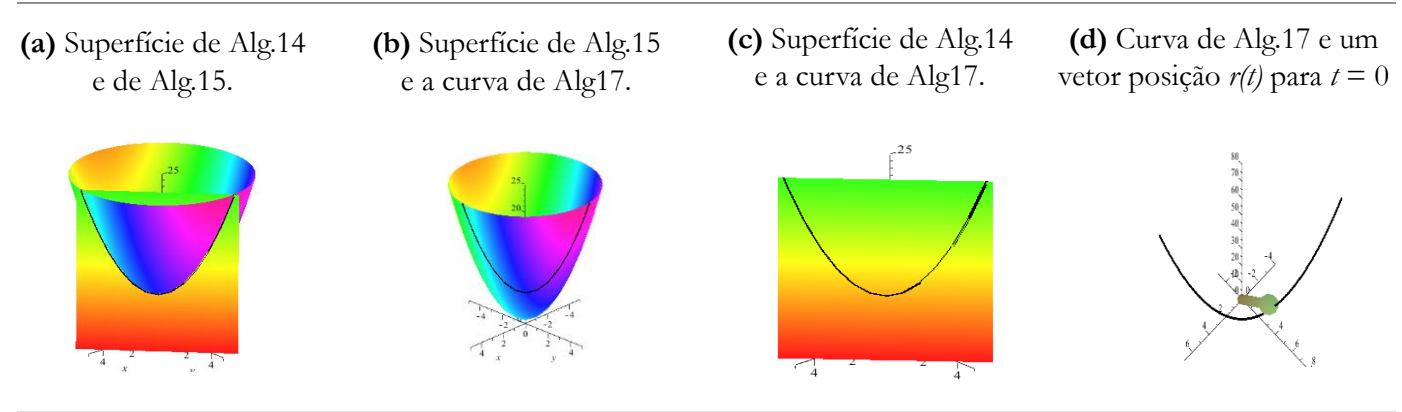

Fonte: Produção dos autores.

Como veremos mais adiante, a realização dessa tarefa, proposta pelo professor de CDI III para os estudantes no ambiente papel/lápis, é um desafio para eles, como mostram os recortes de seus manuscritos no item 6 .

\section{A UESC, o professor de CDI e a análise de práticas de estudantes}

A Universidade Estadual de Santa Cruz (UESC) - Bahia - é uma instituição do Ensino Superior localizada entre dois polos urbanos (Ilhéus e Itabuna) do sul da Bahia, no km 
16 da rodovia Jorge Amado (BR 415), no município de Ilhéus. Ela oferece, entre outros, cinco cursos de Engenharia (Civil, Produção, Elétrica, Mecânica e Química) vinculados ao único Departamento de Ciências Exatas e Tecnológicas (DCET). Dentre as disciplinas reconhecidas nas respectivas grades curriculares, os estudantes desses cursos têm a obrigação de cumprir a matéria CDI, decomposta em três disciplinas CDI I, II e III. Essas disciplinas são propostas com seis créditos $^{8}$ e uma carga horária de noventa horas/aulas cada uma, e são ministradas pelos professores da área de Matemática dessa instituição que tem dois cursos (Bacharelado e Licenciatura). Em todos os semestres letivos são oferecidos os três CDI para todas as Engenharias, a partir dos respectivos colegiados, sendo que uma turma de um colegiado pode conter estudantes matriculados de outros. No segundo semestre do ano letivo de 2014, o primeiro autor deste artigo, como professor da UESC, foi indicado pelo DCET para ministrar a disciplina CDI III no curso de Engenharia de Produção (EP) pela segunda vez consecutiva. Na turma havia 32 estudantes matriculados, inclusive de outras Engenharias, embora predominassem os da EP. Dentre esses, 22 acompanharam a disciplina até ao final do semestre com registros ${ }^{9}$ na Secretaria Acadêmica de cursos de graduação (SECREGE) da UESC. A avaliação apresentada no Quadro 1 é correspondente a um dos seis créditos de CDI III organizados pelo referido professor no segundo semestre de 2014 e aplicada aos 22 estudantes.

Com base nessa avaliação, apresenta-se um diagnóstico (com referência na fase de aplicação, análise a posteriori e validação de uma Sequência Didática - SD, (HENRIQUES, 2011)), sobre as práticas efetivas de estudantes dessa turma, trazendo alguns recortes (cf. Figura 16) de produções de oito dentre os 22 estudantes que realizaram a avaliação, identificados por Estudante-E, $\operatorname{com} E \in[1,8] \subset N$.

Esta escolha se justifica, pois, considerarmos os estudantes que apresentaram menos e os que apresentaram mais informações em suas produções efetivas durante a avaliação.

Na presente análise, levamos em consideração os seguintes, dentre os objetivos de uma SD na mencionada fase, com ênfase no primeiro: (1) compreender e revelar as práticas efetivas de estudantes envolvidos na aplicação da SD (no caso, a avaliação do professor de CDI III) na instituição de referência, (2) validar (confrontar a análise a posteriori com a análise a priorı) e (3) viabilizar as condições de desenvolvimento do objeto de estudo visado, a fim de favorecer a aquisição de conhecimentos significativos dos sujeitos envolvidos.

Optamos por organizar alguns recortes das produções dos estudantes em uma figura. Contudo, no decorrer da análise, poderão surgir recortes complementares que se fizerem necessários.

\footnotetext{
${ }^{8}$ Crédito é um dos sistemas adotados nessa Instituição para creditação de uma disciplina. Assim, "para efeito de registro acadêmico, cada crédito corresponderá a 15 horas/aulas teóricas $(\mathrm{T}), 30$ horas/aulas como prática $(\mathrm{P})$ e 45 horas/aulas de estágio (E)". (Disponível em: <http://www.uesc.br/a_uesc/regimento.pdf>. Acesso em: 16 mar. 2016).

${ }^{9}$ Aqui, o termo registro não é empregado no âmbito da teoria, mas no contexto comum. Ou seja, o "ato de se inscrever em livro próprio a cópia ou extrato de um documento que deve ficar arquivado”. (FERNANDES; LUFT; GUIMARÃES 1990).
} 
Assim, como se pode observar na Figura 16, a maioria dos estudantes avaliados tem certa noção de visualização das superfícies em jogo, no espaço tridimensional, que se manifesta nos esboços das mesmas que eles realizam no registro gráfico, mesmo observando que a noção de registro é evocada de forma errônea. É o caso, por exemplo, em que o Estudante-3, escreve:

A partir das figuras 2 e 3, temos o seguinte registro gráfico da interseção das duas superficies. [Estudante 3].

As Figuras 2 e 3 a que o estudante se refere são esboços, do mesmo, que precedem à sua quarta figura onde esse estudante apresenta o plano e o parabolóide em questão no mesmo sistema de coordenadas, fornecendo, assim, um resultado próximo ao esperado, conforme a análise a priori. Contudo, ele não explicita a referida interseção nesse registro.

Figura 16. Recortes da produção efetiva de estudantes.

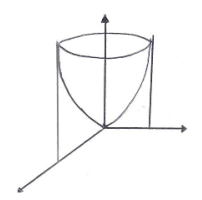

Estudante 1

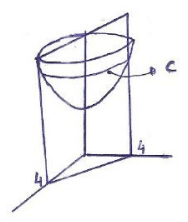

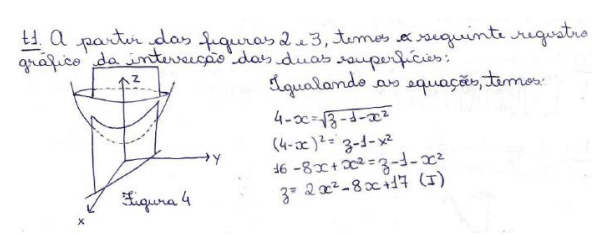

Estudante 3

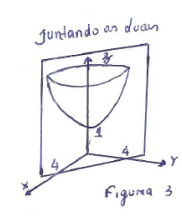

Estudante 4

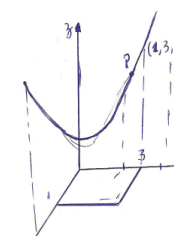

Estudante 5

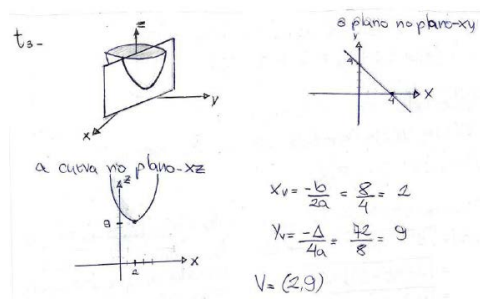

Estudante 6

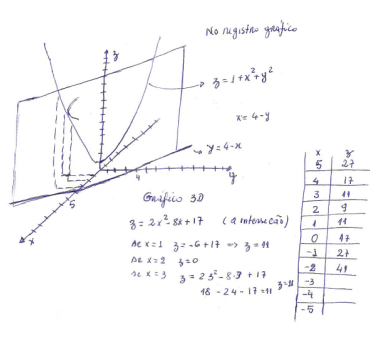

Estudante 7

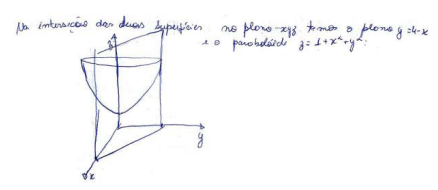

Estudante 8

Fonte: Manuscritos de estudantes do curso de CDI III do referido professor. 
O tratamento que consiste na determinação da curva correspondente no registro algébrico é realizado perfeitamente pelo mesmo Estudante-3, supondo-se que, em uma etapa implícita, de sua prática, ele realizou um cálculo mental para isolar a variável $y$ na equação da superfície parabólica, pois a equação $y= \pm \sqrt{z-1 x^{2}}$ (mesmo com ausência dos sinais \pm ) que ele utiliza no tratamento não é encontrada em parte alguma de seus manuscritos. A Figura 17 ilustra as figuras 2 e 3 mencionadas pelo Estudante-3.

Figura 17. Recorte da produção efetiva do Estudante-3.

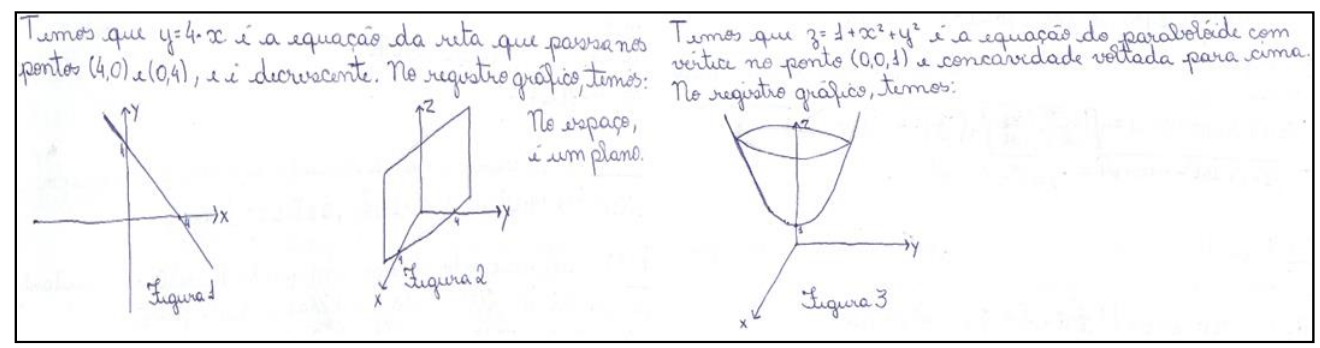

Fonte: Manuscritos de estudantes do curso de CDI III do referido professor.

Se, na descrição da sua Figura 4, o Estudante-3 se confunde com a noção de registro, ele o apresenta como esperado (cf. análise a priori) nas três figuras, quando ele escreve: “... No registro gráfico, temos: [...]", representando, assim, a curva (reta) de equação $z=1+x^{2}+y^{2}$ no plano- $x y$ (figura 1 do estudante), a superfície (plano) dessa mesma equação que contém a referida reta no espaço (figura 2 do estudante). O mesmo ocorre com a representação do parabolóide de equação $z=1+x^{2}+y^{2}$ no registro gráfico (figura 3 do estudante). Mesmo sabendo das limitações proporcionadas pelas técnicas de representação de superfícies no ambiente papel/lápis, pode-se inferir que a produção efetiva do Estudante-3 é validada pelos conceitos desenvolvidos nas análises anteriores.

Sublinha-se que os Estudantes 2, 4, 6 e 8 (cf. recortes na Figura 16) apresentam também uma produção análoga à do Estudante-3, sendo que o tratamento das equações no registro algébrico, favorecendo a descoberta da curva de interseção das superfícies em jogo, não utiliza necessariamente a técnica de comparação na produção desses estudantes. É notável que esses estudantes compreendem bem a ideia de que "intersectar superfícies de equações distintas no registro gráfico significa igualar as equações correspondentes no registro algébrico”, manifestando-se na conversão e na coordenação de representações entre os dois registros. Essa ideia se constitui na técnica dita comparação e é predominante nas suas práticas em detrimento da técnica de substituição, utilizada pelo Estudante-8, como revela o recorte da sua produção na Figura 18. 
Figura 18. Recorte da produção efetiva do Estudante-8.

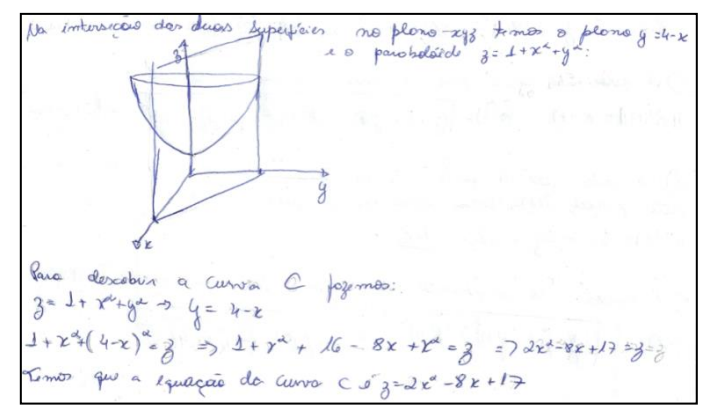

Reprodução do tratamento das equações realizado pelo estudante no registro algébrico.

$z=1+x^{2}+y^{2} \Rightarrow y=4-x$

$1+x^{2}+(4-x)^{2}=z \Rightarrow 1+x^{2}+16-18+x^{2}=z$

$\Rightarrow 2 x^{2}-8 x+17=z$

Temos que a equação da curva C é

$z=2 x^{2}-8 x+17$

Fonte: Manuscritos de estudantes do curso de CDI III do referido professor.

Diferentemente do tratamento de gráficos apresentado pelos cinco estudantes anteriormente citados, baseada na interpretação global de propriedades figurais (DUVAL, 1988), o Estudante-7 apresenta um resultado coberto de dificuldades que se manifesta na representação das superfícies em questão, no registro gráfico. Ele utiliza o procedimento ponto a ponto na tentativa de acessar a visualização dessa superfície no registro gráfico. Por outro lado, utilizando a técnica de substituição, ele fornece a equação da curva de interseção do plano com o parabolóide em questão. Todavia, mesmo tendo em mente a definição de uma função vetorial, embora apresentada de forma incompleta, sem informar o que é $f(t) g(t)$ e $h(t)$, assim como o intervalo do parâmetro $t$, ele se depara com a dificuldade de apresentar as equações paramétricas da curva em questão. A Figura 19 traz um recorte complementar da sua produção durante a avaliação do professor.

Figura 19. Recortes da produção efetiva do Estudante-7.

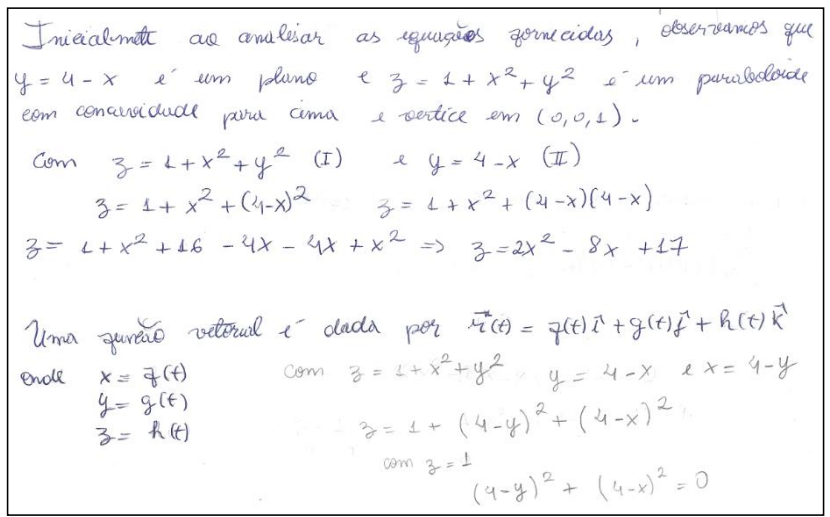

Fonte: Manuscritos de estudantes do curso de CDI III do referido professor. 
De um modo geral, todos os estudantes conseguem encontrar uma equação $z=h(x)$ da curva $\mathbf{C}$ resultante da interseção das duas superfícies com zem função de $x$. Mas não conseguem estabelecer a relação dessa curva com o plano que a contém, bem como com a variável $x$ para decidir sobre o parâmetro $t$ de tempo de variação de um ponto $\mathbf{P}$ sobre a citada curva e, consequentemente, apresentar as equações paramétricas esperadas conforme a análise a priori.

Poder-se-ia afinar a análise e estender a apresentação das práticas efetivas de cada estudante em torno dessa avaliação. Contudo, essa tarefa foi deixada para um trabalho futuro contentando-se com os resultados apresentados aqui. Pode-se ver que alguns estudantes têm certo conhecimento da tarefa proposta, mesmo apresentando dificuldades na conclusão dela e com as limitações impostas pelas técnicas de realização desse tipo de tarefa no ambiente papel/lápis.

Com efeito, acredita-se que a integração de ambientes computacionais de aprendizagem, como o software Maple, nas práticas efetivas em sala de aula, é necessária, por se constituir como recurso auxiliar de aprendizagem dos estudantes. Pois, como visto na análise a priori dessa avaliação, esse ambiente proporciona condições de desenvolvimento do tipo de objetos de estudo envolvidos na referida avaliação; favorece as representações e a visualização de superfícies e curvas no espaço tridimensional.

A nossa experiência, como professores e pesquisadores, tem mostrado a viabilidade dessas condições em sala de aula na Educação Matemática no Ensino Superior.

\section{Considerações finais}

No início deste artigo, apontou-se o propósito de trazer algumas reflexões acerca das aplicações equivocadas de noções relacionadas à Teoria dos Registros de Representação Semiótica por estudantes em situação de aprendizagem, bem como por pesquisadores que a utilizam em suas pesquisas/palestras. Ao longo do artigo, foram apresentadas algumas definições fundadas na proposta de Duval (1993). Elegemos os conceitos de cálculo diferencial e integral (CDI) com ênfase nas funções de duas e três variáveis, como objeto matemático de referência, para aplicar as referidas definições. Com efeito, consideramos uma função de duas variáveis clássica no ensino de CDI para reforçar as três atividades cognitivas fundamentais, ligadas aos registros de representação semiótica (Definições 4 a 6) tornando explícita a compreensão e a apropriação de conceitos e definições, ora apresentadas neste artigo, visando contribuir à reflexão dos demais precursores dessa teoria.

Além disso, para despertar ainda mais o interesse do leitor, inclinamos a atenção nas reflexões sobre as relações possíveis entre os resultados obtidos no estudo dos elementos teóricos e as práticas institucionais desenvolvidas em salas de aulas em uma IES. Para isso, utilizamos uma das avaliações de um professor de Cálculo Diferencial e Integral III da Universidade Estadual de Santa Cruz (UESC), apresentando, assim, um diagnóstico em torno das práticas efetivas de estudantes devidamente matriculados nesta disciplina no segundo semestre de 2014, com o objetivo de compreender e revelar as suas práticas efetivas em confronto (validação) com às análises anteriores, além viabilizar as condições de desenvolvimento do objeto de estudo visado em sala de aula.

Os resultados mostram que é possível trazer contribuições na reflexão e nos modos como a Teoria de Registros de Representação Semiótica está sendo utilizada pelos estudantes 
e pesquisadores nas pesquisas inscritas em Educação, como um todo e particularmente em Educação Matemática, assim como em sala de aula, estendendo-se assim a discussão com os alunos, não apenas sobre os objetos matemáticos específicos, mas principalmente sobre os sistemas ou registros mobilizados para as suas representações.

Espera-se ter trazido contribuições para a análise e o desenvolvimento de pesquisas em Educação Matemática em geral, particularmente no Ensino Superior no que diz respeito à utilização apropriada da Teoria dos Registros de Representação Semiótica (TRRS) e ao Cálculo Diferencial e Integral. As contribuições com este trabalho se inscrevem, portanto, no repensar sobre a utilização eficiente dos elementos da TRRS no discurso do pesquisador, do professor e dos estudantes na Educação Matemática no Ensino Superior, em especial na representação de objetos matemáticos e suas conversões entre registros. Por fim, insiste-se na afirmação sustentada pela Definição 06, de que não se convertem registros, mas as representações de objetos do saber em jogo neles formuladas.

\section{Referências}

BONOTO, D. L.; SOARES, M. A. S.; MARTINS, M. C. M. A análise dos registros de representação semiótica no objeto de aprendizagem "potencializando o seu conhecimento". Vivências, Erechim, v. 6, n. 9, p.13-24, 2010. Disponível em: <http:/ /www.reitoria.uri. br/ vivencias/Numero_009/artigos/artigos_vivencias_09/n9_1.pdf $>$. Acesso em: 16 mar. 2016.

CHEVALLARD, Y. L'analyse des pratiques enseignantes en théorie anthropologique du didactique. Recherche en Didactique des Mathématiques, Grenoble, v. 19, n. 2, p. 221 266, 1999.

. Concepts fondamentaux de la didactique : perspectives apportées par une approche anthropologique. Recherches en Didactique des Mathématiques, Grenoble, v. 12, n. 1, p. 73-112, 1992.

DELGADO, C. J. B. O ensino da função afim a partir dos registros de representação semiótica. 2010. 153 f. Dissertação (Mestrado em Ensino das Ciências na Educação Básica) - Universidade do Grande Rio "Prof. José de Souza Herdy", Duque de Caxias, 2010. Disponível em: < http://www2.unigranrio.br/unidades_adm/pro_reitorias/propep/stricto_ sensu.old/cursos/mestrado/ensino_ciencias/galleries/downloads/dissertacoes/dissertacao_ carlos_jose_borges_delgado.pdf >. Acesso em: 16 mar. 2016.

DUVAL, R. Graphiques et équations: l'articulation de deux registres. Annales de

Didactiques et de Sciences Cognitives, Strasbourg, v. 1, p. 235-253, 1988.

Registres de représentation sémiotique et fonctionnement cognitif de la pensée.

Annales de Didactiques et de Sciences Cognitives, Strasbourg, v. 5, p. 35-65, 1993.

. Sémiosis et pensée humaine. Bern: Peter Lang, 1995. 
DUVAL, R. Signe et objet (I): trois grandes étapes dans la problématique des rapports entre représentation et objet. Annales de Didactiques et de Sciences Cognitives, Strasbourg, v. 6, p. 139-163, 1998.

FERNANDES, F.; LUFT, C. P.; GUIMARÃES, F. M. (Org.). Dicionário brasileiro Globo. 16. ed. Rio de Janeiro: Globo, 1990.

HENRIQUES, A. L'enseignement et l'apprentissage des intégrales multiples: analyse didactique intégrant l'usage du logiciel Maple. Grenoble: Lab. Leibniz, 2006.

Reflexões sobre análises institucionais e seqüências didáticas: o caso do estudo de integrais múltiplas. Ilhéus: UESC, 2011. Disponível em: <https://sites.google. com/site/gpemac/dissertacoes-de-mestrado>. Acesso em: 30 set. 2015.

HENRIQUES, A.; NAGAMINE, A.; NAGAMINE, C. M. L. Reflexões sobre análise institucional: o caso do ensino e aprendizagem de integrais múltiplas. Bolema, Rio Claro, v. 26, n. 44, p. 1261-1288, 2012.

PEIRCE, C. S. Semiótica. São Paulo: Perspectiva, 2005.

SILVA. J. R. D. Um estudo de registros de representação semiótica na aprendizagem dos conceitos de máximos e mínimos de funções. 2005. 120 f. Dissertação (Mestrado em Educação) - Universidade Federal de Mato Grosso do Sul, Campo Grande, 2005. Disponível em: <http://repositorio.cbc.ufms.br:8080/jspui/handle/123456789/739>. Acesso em: 16 mar. 2016.

SWOKOWSKI, E. W. Cálculo com geometria analítica. 2. ed. São Paulo: Makron Books, 1994. v. 2.

VERTUAN, R. E.; SILVA, K. A. P.; ALMEIDA, L. M. W. O registro gráfico em atividades de modelagem matemática: um estudo da conversão entre registros segundo a teoria dos registros de representação semiótica. In: SEMINÁRIO INTERNACIONAL DE EDUCAÇÃO MATEMÁTICA, 2., 2009, São Paulo. Disponível em: <http://www.uel.br/ grupo-pesquisa/grupemat/docs/CC01_siemat2009.pdf>. Acesso em: 16 mar. 2016. 
